\title{
Collaborative oral health education for caregivers in an assisted-living facility
}

\author{
Susan Parsons \\ School of Nursing, Wichita State University, Kansas, USA \\ Correspondence: Susan Parsons. Address: School of Nursing, Wichita State University, 1845 Fairmount, Wichita, Kansas \\ 67260-0041, USA. Email: susan.parsons@wichita.edu.
}

Received: J une 19, 2012

DOI : $10.5430 /$ jnep.v3n3p44

\section{Abstract}

Background: The purpose of this study was to implement a collaborative oral health project and evaluate its effectiveness for caregivers in an assisted-living facility. An interdisciplinary study was conducted by nursing and dental hygiene faculty.

Methods: A quasi-experimental design was utilized with a pre-post treatment format. Pre-and post-treatment scores were analyzed from a convenience sample of 13 caregivers in an assisted-living facility. The information addressed oral care assessments, interventions, evaluation and referral guidelines.

Results: Post-treatment scores were significantly higher than pre-treatment scores $(p=.002)$. The results indicate the collaborative teaching project was effective increasing knowledge of assisted-living caregivers regarding oral hygiene practices for older adults.

Conclusions: Based on these findings the results indicate collaborative oral health care teaching project was effective in increasing caregiver oral hygiene knowledge. It was concluded that the collaborative instruction was effective. Other considerations included positive outcomes on a follow-up survey assessment of caregivers and assisted-living management regarding utilization of new information that had been presented.

\section{Key words}

Collaboration, Oral health care, Caregivers, Assisted-living

\section{I ntroduction}

Recent research studies indicate that poor oral hygiene correlates with an increase in some systemic diseases such as aspiration pneumonia and cardiovascular disease ${ }^{[1-4]}$. Poor oral health is increasingly recognized as a link to other diseases. Lack of caregiver knowledge and education about oral care is associated with increased lack of optimal oral health. Elderly patients residing in long-term care facilities reportedly are particularly vulnerable ${ }^{[5]}$. Recent findings indicate why daily oral care is essential for overall health.

A significant association has been found between the presence of pathogenic oral bacteria and the incidence of aspiration pneumonia ${ }^{[2]}$. Ishikawa et al. ${ }^{[1]}$ documented that levels of oropharyngeal bacteria decreased or disappeared after consistent professional oral health care, including cleaning of teeth, dentures, tongue, and oral mucous membranes. 
Mechanical cleaning of the oral cavity is an important strategy to prevent aspiration pneumonia ${ }^{[1]}$. Oral care can also improve cough reflex sensitivity in elderly nursing home patients which may reduce the likelihood of aspiration ${ }^{[3]}$. As a result, professional oral health care is effective in reducing respiratory pathogens ${ }^{[4]}$.

A major study involving 4,254 participants revealed cardiovascular complications due to poor oral health. Periodontal disease and the number of remaining teeth are related to the prevalence of myocardial infarction and hypertension ${ }^{[6]}$. Participants with severe periodontal disease had a 4.3 times greater risk of stroke than the control participants ${ }^{[7]}$. Periodontal disease was also found to adversely affect glycemic control in diabetic patients ${ }^{[8]}$.

In a statement before the United States Senate Special Committee on Aging, “Ageism in Healthcare: Are Our Nation's Seniors Receiving Proper Oral Health Care?” (Sept. 22, 2003), Dr. Richard Carmona, Surgeon General of the United States, said "The burden of oral infections and conditions that affect the mouth, face, and jaw are so broad and extensive that the dentists can't do it alone." ${ }^{[9]}$ It will take all of us working together to continue to make progress in advancing the oral health of all Americans. In a 2003 report of the Surgeon General (Oral Health in America) Carmona discussed findings which indicated oral health is essential to general health and well-being ${ }^{[9]}$. Older adults are more prone to chronic, disabling diseases and conditions. Problems with daily medications, which often have an adverse effect on their oral health, are more likely. The World Health Organization (WHO) recommends that countries adopt strategies for improving oral health care of elderly individuals ${ }^{[10]}$.

Stein and Henry ${ }^{[5]}$ emphasized that nurses must provide better oral care to older adults and patients with severe disabilities. It has been recommended that nursing and dental hygiene schools collaborate to provide appropriate instruction to nurses and nurse assistants who potentially may provide oral care to this vulnerable population ${ }^{\text {[11] }}$. Collaboration between health professionals has been a successful strategy for improving outcomes.

Curriculum needs to cover oral examinations, pathology, adverse oral effects of drugs, guidelines for prevention, and referrals. Information should be integrated with other common topics such as health assessment, nutrition, and palliative care ${ }^{[11]}$. Content of educational programs has also been shown to improve nursing assistant attitudes toward oral care ${ }^{[12]}$. Successful educational programs include:

- Instruction on the importance of oral care

- Instruction on assessment ${ }^{[13]}$

- Daily oral care documentation forms ${ }^{[14]}$

- Oral disease education

- Provision of oral care hygiene supplies ${ }^{[15,16]}$

- Education of small groups of caregivers ${ }^{[17]}$

- Hands-on education for delivery of oral care ${ }^{[18]}$

Interdisciplinary collaboration is necessary to promote oral health care among older individuals ${ }^{[19]}$. The state of collaborative oral health care in previous studies show opportunities for a variety of health care providers to assist in improved oral care outcomes for long-term care residents. Nurses and nurse assistants can provide oral care for residents visually observing the mouth on a daily basis. Physicians, dietitians, social workers, occupational therapists and allied health professionals observe residents and are in a position to report concerns to the nurses who can refer to dental hygienists and dentists. Thus, different healthcare professionals could collaborate on interprofessional teams to promote oral hygiene of older adults in institutional facilities ${ }^{[20]}$. Nurses may consider oral care important, yet, may not have a knowledge of the oral care link to overall health. Educating nurses about oral health has been inadequate regarding the 
interrelationship between oral health and overall health ${ }^{[21]}$. Also, the oral care given by caregivers may not be performed effectively due to an inadequate level of oral care knowledge ${ }^{[20]}$.

A recent large 17 year cohort study of over 5,000 older men and women showed a decreased risk of mortality with several dental behaviors, number of teeth, and dentures suggesting that maintenance of good oral hygiene is an important health promoter in the aging population ${ }^{[22]}$.

Although educational programs have been implemented in nursing homes, published research regarding assisted-living facilities is generally lacking. This factor led to an investigation that targeted an assisted-living facility. A collaboration involved nursing and dental hygiene faculty to promote an increased awareness of the importance of daily oral care. Techniques for optimal oral care were implemented.

\section{Methods}

The purpose of this study was to implement a collaborative oral health project and its effectiveness for caregivers in an assisted-living facility. Nursing and dental hygiene faculty presented information on the integration between oral health care and systemic diseases, proper nutrition, and medication affecting dry mouth. Dental hygiene faculty presented proper oral hygiene assessment, interventions, techniques, evaluation, and referrals. Implementation of proper care followed. Penlights were provided to assist in examining the mouth. Specialized toothbrushes, dental floss, and hand sanitizers were dispensed to assist with oral care. Specialized toothbrushes had a double-head of bristles which allowed the resident or caregiver to cover both sides of the teeth simultaneously. Less effort is required to brush teeth for older adults with disabilities or neuromuscular disorders utilizing specialized toothbrushes ${ }^{[20]}$.

A quasi-experimental design was utilized. Identical 20 question pre-and post- treatment questionnaires were administered to the caregivers. In addition three optional open-ended questions included on the post-treatment questionnaire were: 1) What have you particularly learned from this information? 2) What additional information would be helpful during this educational session? 3. What are you planning to do differently after learning the oral health information?

Pre- and post-treatment scores were analyzed from a sample of assisted-living caregivers.

\subsection{Setting}

This study was conducted at an assisted-living nursing facility with a capacity of 48 residents. Internal Review Board (IRB) approval was obtained from an urban university prior to the presentation. Participation was voluntary, and caregivers were informed that individual answers would remain confidential.

\subsection{Sample}

An educational program with a pre- and post-treatment format for 13 caregivers, including one Registered Nurse (RN), two Licensed Practical Nurses (LPNs), six Certified Nursing Assistants (CNAs), two dietary staff members, and one Social Service member $(n=13)$, were included. The age categories of the participants reported were: under age $30(n=4)$; age 30-40 (n=1); age 40-50 (n=2); and age 50 and over $(n=2)$; no data $(n=4)$.

Although dietary and social service participants did not provide direct mouth care, they interact verbally with the residents and have access to promote oral health care by asking questions regarding ability to eat and potential pain with eating.

\subsection{Description of program}

A specialized oral instructional program workbook, developed by Specialized Care Company, in part, via a grant from the National Institute of Dental Research, was adapted for the pre-post questionnaire ${ }^{[23]}$. The workbook was designed in 
collaboration with dental experts specifically to be used by people in community residences who are responsible for daily oral hygiene of the residents. The goal was to provide an instructional program to help participants appreciate the importance of dental care for everyone, including themselves as well as the people they serve ${ }^{[20]}$. The link between poor oral care and systemic health problems highlights the importance of dental care for everyone. The program consisted of six modules including:

- $\quad$ Getting Involved and Overcoming Obstacles

- $\quad$ Good Oral Care

- $\quad$ Tricks of the Trade

- You are What You Eat

- $\quad$ Oral Care for People Missing Teeth

- Preparing for a Dental Visit with a Client

Twenty essential questions regarding oral care were selected from the six modules by the nurse/dental hygienist collaborators for the pre/post treatment questionnaire.

The Oral Health Care Pre-Post Treatment Questionnaire included: true/false and multiple choice questions about proper tooth brushing and flossing techniques, cavity and acid attack prevention, effect of medications to teeth, food which contain sugars harmful to teeth, relationship of food textures to health teeth, importance of regular dental care for everyone especially for those with disabilities, indications of a dental problem causing pain, saliva's cavity fighting abilities, checking the mouth for serious dental problems, and denture care.

\subsection{Procedures}

A pre-treatment questionnaire was administered prior to the educational presentation to assess each caregiver's knowledge regarding oral health. Next, a 1-hour collaborative teaching program included a PowerPoint presentation on oral health care for older individuals. The collaborative educational program information also included procedures for best practices $^{[24,25]}$. An example of instruction by either the nursing faculty, dental hygiene faculty or both included:

- Importance of proper oral health care

- Normal/abnormal physiology of the mouth and gums

- Frequency of oral care

- $\quad$ Proper use of toothbrush and flossing

- Effect of medications on teeth

- Problems related to xerostomia (dry mouth)

- Interventions for patients with disabilities presented

- Nutritional impact

- Potential oral care problems relating to aging

- When to refer

A 20 question post-treatment questionnaire, identical to the pre-treatment questionnaire, was administered to assess knowledge learned from the educational program. 


\section{Analysis and results}

Data were entered and analyzed by the SPSS statistical package for Windows. Repeated-measure $t$-tests were completed to compare pre-treatment scores and post-treatment scores for the caregiver group.

Knowledge differences were assessed by comparing pre- and post- instruction questionnaire scores of the participants. Post-instruction scores $(\mathrm{M}=16.77, \mathrm{SD}=2.28)$ were significantly higher than pre-instruction scores $(\mathrm{M}=14.69$, SD = 2.63), $t(12)=3.96, p=.002$, two-tailed. The increase between means, which was 2.08 points (SD = 1.89), with a confidence interval (95\%) 0.93 points to 3.22 points, supports the premise that the collaborative oral health presentation for caregivers was effective.

Three optional questions on the questionnaire were included.

1) What have you particularly learned from this information?

Answers given by caregivers included: they had learned poor oral hygiene can cause health issues like pneumonia; helping elderly individuals keep good hygiene with flossing and brushing is just as important as for a younger person; the importance of cleaning teeth and a healthy mouth; and how to maintain teeth and gums.

2) What additional information would be helpful during this educational session?

Answers included: additional helpful information would include the area regarding non-insured adults/children.

3) What are you planning to do differently after learning the oral health information?

After learning the oral health information caregiver plans included: investing more time to oral hygiene for themselves and people they care for including their children; helping residents to brush more often oral at least rinse teeth after meals; brushing longer than 1 minute; flossing teeth daily and always; making sure the toothpaste contains fluoride, and the importance of training staff.

A follow-up survey assessment included a self-reported Caregiver Survey. The Caregiver Survey was conducted to evaluate utilization of new information presented at the collaborative oral health care presentation. Although the usual time frame to assess for a follow-up survey is at least 6 months, data were collected from the same caregivers who were still available 7.5 months later. Outcomes were requested concerning caregiver implementation of information from the initial oral health care educational presentation. The primary investigator and management director met with caregivers to receive the surveys and answer any questions.

Thirteen caregiver self-reported surveys were received, which provided information regarding new information utilization following the initial educational project which included:

1) Utilization of oral hygiene products used for oral care of residents included a penlight, toothpaste, dental floss holders, hand sanitizer, dental floss, double-headed toothbrushes.

2) Referral to an informational folder to help with oral health care of residents included gum disease, cancer, dry mouth, medication information tips, and tricks for oral care.

3) Teaching residents the importance of oral health care included brushing, mouth rinse, proper nutrition, flossing, and other.

4) Important perceived information recorded following the presentation utilized included tips on how to assist with brushing teeth and that oral care is imperative. Other comments included giving residents the opportunity for good oral care and how not taking care of one's teeth affects overall health. 
5) Improvement in the oral health care of residents included fresher breath, less dry mouths, fewer eating problems, less bleeding gums, fewer complaints of pain.

A Self-Reported Assisted-Living Manager Survey revealed:

1) Management did not respond regarding where there were fewer cases of pneumonia in residents because the prevalence of community-acquired pneumonia is usually between September and March ${ }^{[26]}$. No cases of aspiration pneumonia were reported.

2) Oral health care practices/procedures changed regarding oral health care following the Collaborative Oral Health Care presentation as two residents had been referred to a Dentist.

3) The most information learned from the presentation reported pertained to bad breath and brushing the entire mouth, including the gums.

4) The gift to management a Specialized Care-Oral Health Care DVD for Caregivers had been utilized twice for orientation of new caregivers.

5) Other comments included: Bad breath led Certified Nurse Assistants (CNAs) to brush the roof of mouth, tongue, etc., when doing they found that some residents had loose and/or decayed teeth. As a result, referrals were made to dentists, and two residents received dentures.

\section{Discussion}

The purpose of this study was to develop and implement a collaborative instructional project on oral health care of residents presented to caregivers in an assisted-living facility. An evaluation of its effectiveness for caregivers yielded positive outcomes including significant gains in knowledge from the post- instruction questionnaire. A follow-up self-reported caregiver questionnaire indicated caregivers had utilized the educational material and oral care equipment given to them during the project. Residents were referred to a dentist when oral care problems were noted. Nursing and dental hygiene faculty members combined their educational resources to promote the importance of oral health care in older residents and teach caregivers optimal oral health care principles. Results of this study are in agreement with results obtained from a few prior studies that also indicated nurses and nurse aids can make a difference in the oral health of residents ${ }^{[28]}$. Oral assessment and hygiene have an impact on nutritional intake and can affect the quality of life ${ }^{[28]}$.

It was stressed that asking two basic questions: (1) “Can you eat?” and (2) “Do you have any pain?” each day for the individual residents is vital for an accurate caregiver's assessment and referral. Dental professionals must make an effort to inform medical doctors, nurses, and caregivers of dependent elderly individuals regarding the effectiveness of oral health care. Nurses working in long-term care are in the best position to include the mouth in their assessments and refer patients to dental professionals for follow-up ${ }^{[28]}$. Better oral health care is necessary. Nursing and dental hygiene collaboration hold promise for improving oral health for institutionalized older adults ${ }^{[28]}$.

\section{Suggestions for further research}

This research represents a step toward nursing and dental hygiene collaborative education for assisted-living caregivers. Future studies with a larger sample size are recommended. A long-term term goal is to improve oral hygiene in assisted-living care settings. It is likely that there would be a decreased number of potential problems such as pneumonia, myocardial infarctions, stroke, and diabetes. Collaborative/interdisciplinary research most likely will benefit oral health care in assisted-living as well as and long-term care facilities. 


\section{I mplications and conclusions}

Knowledge of proper oral health care in older adults by caregivers is essential to prevent unnecessary health complications. Oral problems in older individuals can lead to poor general health and pain. When older adults enter assisted-living facilities, the potential for oral health problems and systemic illnesses increase. A collaborative education program presented by nursing and dental hygiene educators can effectively increase the knowledge level of caregivers in assisted-living facilities. Providing combined information from both nursing and dental hygiene faculty regarding techniques of good oral health assessment and information about inflammation links to general poor health are essential.

Even though the sample size was small, this research adds to the body of knowledge regarding the importance of providing oral health education to caregivers of elderly individuals. Residents and caregivers can increase their awareness and knowledge of the importance of good oral hygiene. Proper oral health equipment can effectively increase the quality of the caregivers' work. Caregivers with increased information concerning oral health assessments, interventions, and when to refer to prevent complications will have the potential to improve oral health in older adults residing in assisted-living facilities.

\section{Acknowledgement}

I would like to acknowledge and thank WSU Dental Hygiene Clinical Educator, Dawn McGlasson, RDH, MPH for assistance with collaborative oral health education, Dr. Barbara Hodson for project review and Dr. Doug Parham for statistical project support.

\section{References}

[1] Ishikawa A, Yoneyama, T, Hirata, K, Miake, Y, Miyatake, K. Professional oral health care reduces the number of oropharyngeal bacteria. Journal of Dental Residents. 2008; 8(6): 594-598. http://dx.doi.org/10.1177/154405910808700602

[2] Terpenning M, Taylor, M, Ohrui, T, Mukaiyama, H, Okamoto, H, Hoshiba, K, et al. Aspiration pneumonia: Dental and oral risk factors in an older veteran population. Journal of American Geriatric Society. 2001; 49(5): 557-63. http://dx.doi.org/10.1046/j.1532-5415.2001.49113.x

[3] Watando A, Ebihra S, Ebihara, T, Okazaki T, Takahashi T, Asda M., et al. Daily oral care and cough reflex sensitivity in elderly nursing home patients. Chest. 2004; 126(4): 1066-1070. PMid:15486365 http://dx.doi.org/10.1378/chest.126.4.1066

[4] Yoneyama T Yoshida, Ohrui, T, Mukaiyama, H, Okamoto H, Hoshiba K, et al. Oral Care reduces pneumonia in older patients in nursing homes. Journal of American Geriatric Society. 2002; 50(3): 430-3. http://dx.doi.org/10.1046/j.1532-5415.2002.50106.x

[5] Stein P, Henry R. Poor oral hygiene in long-term care. American Journal of Nursing. 2009; 109(6): 44-50. PMid:19478605 http://dx.doi.org/10.1097/01.NAJ.0000352472.70993.3b

[6] Holmlund A, Holm G, Lind L. Severity of periodontal disease and number of remaining teeth are related to the prevalence of myocardial infarction and hypertension in a study based on 4,254 subjects. Journal of Periodontology. 2006; 77(7): 1173-8. PMid:16805679 http://dx.doi.org/10.1902/jop.2006.050233

[7] Grau A, Becher, H, Ziegler, C., Lichy, C, Buggle, F, Kaiser, C., et al. Periodontal disease as a risk factor for ischemic stroke. Stroke. 2004; 35(2): 496-501. PMid:14707235 http://dx.doi.org/10.1161/01.STR.0000110789.20526.9D

[8] Taylor G, Borgnakke W. Periodontal disease: associations with diabetes, glycemic control and complications. Oral Disease. 2008; 14(3): 191-203. PMid:18336370 http://dx.doi.org/10.1111/j.1601-0825.2008.01442.x

[9] Carmona R. Ageism in healthcare: Are our nation's seniors receiving proper oral health care? Office of the Surgeon General. Sept. 22, 2003.

[10] Petersen PE, Yamamoto T. Improving the oral health of older people: the approach of the WHO Global Oral Health Programme. Community Dental Oral Epidemiology. 2005; 33: 81-92. PMid:15725170 http://dx.doi.org/10.1111/j.1600-0528.2004.00219.x

[11] Coleman P. Opportunities for nursing-dental collaboration: Addressing oral health needs among the elderly. Nursing Outlook, 2005; 53(1): 33-9. PMid:15761398 http://dx.doi.org/10.1016/j.outlook.2004.06.008

[12] Coleman P. Promoting oral health in elder care; challenges and opportunities. Journal of Gerontological Nursing. 2004 ; $30(4): 3$. PMid:15109040

[13] Malkin B. The importance of patients' oral health and nurses’ role in assessing and maintaining it [Internet]. 2009 ; 105 (17): 19. Available from: http://www.nursingtimes.net (18 June, 2012, date last accessed). 
[14] Charteris P, Kinsella T. The oral care link nurse: A facilitator and educator for maintaining oral health for patients at the Royal Hospital for Neuro-disability. Special Care Dentistry. 2001; 21(2): 68-71. http://dx.doi.org/10.1111/j.1754-4505.2001.tb00228.x

[15] Boczko F, McKeon S, McHugh S, Bagg J. Long-term care and oral health knowledge. Journal of American Medical Directors Association. March 2009; 204-206. PMid:19233061 http://dx.doi.org/10.1016/j.jamda.2008.08.007

[16] Frenkel H, Harvey I, Newcombe R. Improving oral health in institutionalized elderly people by educating caregivers: a randomized controlled trial. Community Dental Oral Epidemiology. 2001; 29(6): 289-97. http://dx.doi.org/10.1016/j.jamda.2008.08.007

[17] Nicol R, Sweeny M, McHugh S, Bagg, J. Effectiveness of health care worker training on the oral health of elderly residents of nursing homes. Community Dental Oral Epidemiology. 2005; 33: 115-24. PMid:15725174 http://dx.doi.org/10.1111/j.1600-0528.2004.00212.x

[18] Jablonski, RA. Examining oral health in nursing home residents and overcoming mouth care-resistive behaviors. ISSN: 1524-7929. 2010; 18(1): 21-26.

[19] Baily R, Gueldner, S, Ledikwe, J, Smicklas-Wright, H. The oral health of older adults: An interdisciplinary mandate. Journal of Gerontological Nursing. 31 (7), 11-7.

[20] Yoon MN, Steele CM. The oral care imperative: the link between oral hygiene and aspiration pneumonia. Topics in Geriatric Rehabilitation. 2007; 23(3): 280-8.

[21] Dolce M, Nurse Faculty Enrichment and Competency Development in Oral-Systemic Health. Nursing Research and Practice; 2012. http://dx.doi.org/10.1155/2012/567058.

[22] Paganini-Hill A, White S, Atchison, K. Dental Health Behavior, Dentition, and Mortality in the Elderly: The Leisure World Cohort Study; Journal of Aging Research. 2011; http://dx.doi.org/10.4061/2011/156061.

[23] Specialized Care Co. Hampton [Internet]. NH. 2006; Available from: http://www.specializedcare.com (18 June 2012, date last accessed).

[24] Hartford Institute for Geriatric Nursing [Internet]. 2010. Available from: http://consultgerirn.org (18 June 2012, date last accessed).

[25] National Institute of Dental and Craniofacial Research [Internet]. 2009. Available from: http://www.nidcr.nih.gov/oralhealth (18 June 2012, date last accessed).

[26] Sinai-Grace Hospital. Community acquired pneumonia [Internet]. 2009; Detroit, Michigan. Available from: http://www.sinaigrace.org/id=537 (7 April, 2012, date last accessed).

[27] Vargas C, Kramarow E, Yellowitz, J. The oral health of older Americans. 2001; Hyattsville, MD.: National Center for Health Statistics, 1-7.

[28] Jablonski RS, Swecker T, Munro C, Grap M, Ligon M. Measuring the Oral Health of Nursing Home Elders. Clinical Nursing Research. 2009; 18 (3): 200-21. PMid:19386817 http://dx.doi.org/10.1177/1054773809335306 